also illustrated some of the difficulties in attempting comparisons of different amino acid solutions.

We have previously drawn attention to abnormal plasma amino acid profiles in Vamin 9 fed infants. ${ }^{2}$ We considered this to be a reflection of the amino acid composition of Vamin 9 rather than an idiosyncratic reaction, as the pattern recurred consistently, and was much less evident when a modified solution containing less phenylalanine was used. ${ }^{3}$ One factor which McIntosh and Mitchell do not address in detail, but which may have considerable influence on plasma amino acid profiles, is the amount of enteral nutrition being given. In our original index case of hyperphenylalaninaemia, ${ }^{2}$ small volumes of breast milk given as a supplement to parenteral nutrition produced large changes in plasma phenylalanine. The patients of McIntosh and Mitchell received either breast milk or preterm formula in addition to their intravenous nutrition. In fact, by day 5 of the study, the only day during parenteral nutrition on which plasma amino acid profiles were measured, the figures given for mean enteral feed in both infants receiving Vamin 9 and those fed with MB233G represent approximately $60 \%$ of the total nitrogen intake. It is therefore difficult to see how valid conclusions can be drawn about the suitability of either amino acid solution as a parenteral nitrogen source.

Although 68 patients were recruited to the study, some died, some were excluded, and some had inadequate blood sampling or blood taken on the wrong day. As a result, the data derive solely from a single plasma amino acid analysis, performed on only seven patients in the Vamin 9 group and five in the MB233G group. Moreover, individual amino acid concentrations are reported as mean (SD) suggesting normal distribution of data. However, the fact that $-2 S D$ would give a negative value for $50 \%$ of the amino acids measured indicates non-parametric distribution of results, consistent with similar studies. ${ }^{3}$ It would therefore be interesting to be able to compare mean (after log transformation to 'normalise' the data) and ranges of individual amino acids.

Finally, single measurements of plasma amino acid profiles may not allow adequate comparison of different solutions as plasma concentrations can vary considerably. This is illustrated by the following plasma concentrations of phenylalanine and tyrosine measured every two weeks in a child with gastroschisis receiving total parenteral nutrition with a constant nitrogen intake: phenylalanine 149,500 , $200,49,63,983$, and $260 \mu \mathrm{mol} / \mathrm{l}$ and tyrosine $95,60,80,58,86,86$, and $145 \mu \mathrm{mol} / 1$.

The theoretical basis for the formulation of MB233G is of interest, but it remains to be seen whether or not this new solution offers any advantages over alternative preparations.

J W L PUNTIS University Department of Paediatrics and Child Health,
Infirmary, Leeds LS2 9NS

I W BOOTH Institute of Child Health, niversity of Birmingham,

1 McIntosh N, Mitchell V. A clinical trial of two parenteral nutrition solution

2 Puntis JWL, Edwards MA, Green A, Morgan I, Booth IW, Ball PA. Hyperphenylalaninaemia in parenterally

3 Puntis JWL, Ball PA, Preece MA, Green A Brown GA, Booth IW. Egg and breast mill based nitrogen sources compared. Arch Dis Child 1989;64:1472-7.
Professor McIntosh comments:

Drs Puntis and Booth suggest that the entera component of nutrition may be important in the development of hyperphenylalaninaemia. They point out that on day 5 both groups of infants in our study received approximately $60 \%$ of their nitrogen intake enterally. We agree with this, as at this time the intravenous nitrogen intake was about $40 \%$ of the total! The point of difference in our two groups was the composition of the intravenous amino acid mixtures and it would seem therefore to be a 'non-sequitur' to suggest the high concentrations of phenylalanine, tyrosine, serine, proline, and asparagine were due to the enteral intake.

There were in fact 16 and 14 patients with 'good' samples for amino acid analysis taken on day 5-see original table 5. We accept that the results were not in a Gaussian distributionthis is why Wilcoxon's signed rank test was used when comparing amino acid concentrations the two groups.

We note the information in the last paragraph which we find very interesting.

We believe that the apparent ability of babies given MB233 to keep their plasma aminogram in the reference range of cord blood aminograms makes it an attractive alternative to Vamin 9 glucose where the aminograms at five days approached the values of infants with untreated phenylketonuria and hereditary tyrosinaemia.

\section{Virological investigations of acute encephalopathy in India}

SIR,-Rabies should be considered in the differential diagnosis of acute encephalopathy in children living in endemic areas. It may present as a non-specific encephalitis without the pathognomonic features such as hydrophobia and a clear history of exposure is not always present. It was therefore surprising that it received no mention in the series of Kumar et $a l^{1}$-could it have accounted for some of the cases among the $40 \%$ in whom they found no cause?

SUSAN M HALL

PHLS Communicable Disease Surveillance Centre, 61 Colindale Avenue,
London NW9 SEQ

1 Kumar R, Mathur A, Kumar A, Sethi GD, gharma S, Chaturvedi UC. Virological investiDis Child 1990;65:1227-30.

Dr Kumar comments:

Rabies is endemic in the Indian subcontinent. It produces an acute encephalitic illness usually with a pronounced psychological component. We agree that occasionally none of these pathognomonic features may be found and therefore it may account for a few of the fata 'unexplained encephalopathies' in this study. Although we have discussed other viral encephitides as a cause in this group we have not specifically mentioned rabies. We therefore appreciate Dr Hall's comment.

SIR,-Dr Kumar and his colleagues in reporting their investigations into the cause of acute encephalopathy in children in Lucknow have not considered the possibility of cerebral cysticercosis in their differential diagnosis. ${ }^{1}$
Cysticercosis in India is not uncommon ${ }^{2}$ and parenchymal involvement, the most common form in children, ${ }^{3}$ may present acutely. Acute diffuse parenchymatous disease presents with generalised cerebral oedema often severe enough to cause an acute rise in intracranial pressure with deterioration of consciousness and cerebral shifts. Acute focal parenchymal disease presents with localised patchy oedema often resulting in convulsions.

The diagnosis of cerebral cysticercosis may present difficulties. Serology is beset with the problems of poor sensitivity and specificity and cerebrospinal fluid changes are often nonspecific and non-diagnostic. Computed tomography has improved diagnostic accuracy but it not always readily available in India. Where there has been the opportunity to perform enhanced computed tomography on Indian patients presenting with seizures, lesions suggestive of cysticercosis have been reported in $26 \%$ of cases, ${ }^{4}$ excision biopsy confirming the diagnosis in a further series of cases. ${ }^{5}$

A diagnosis of cerebral cysticercosis should be considered in all children presenting with 'acute encephalopathy' in India.

B J HEAP
Royal Army Medical College,
Millbank, London SWIP $4 R \mathcal{R}$

1 Kumar R, Mathur A, Kumar A, Sethi GD, Sharma S, Chaturvedi UC. Virological investigations of acute encephalopathy in India. Arch Dis Child 1990;65:1227-30.

2 Balasubramaniam V, Kanaka TS, Ramamurth B. Cerebral cysticercosis in India. Int Surg 1971;56:172-81.

3 Mervis B, Lotz JW. Computed tomography in parenchymatous cerebral cysticercosis. Clin Radiol 1980;31:521-8.

4 Wadia RS, Makhale CN, Kelkar AV, Grant KB Focal epilepsy in India with special reference to lesions showing ring or disc-like enhancement lesions showing ring or disc-like enhancement on contrast computed tomography. F N

5 Chandy MJ, Rajshekhar V, Prakash S, et al.

Cysticercosis causing single, small CT lesions in Indian patients with seizures. Lancet 1989;i: 390-1.

Auditing community screening for undescended testes

SIR,-With interest we read the article of Tamhne et al about the screening for undescended testes. We were surprised by reported high cumulative rates of orchidopexy especially as the authors state that some of the children in the younger cohort would not have their undescended testes detected and operated on.

In The Netherlands during the period from 1976 to 1986 about $3 \%$ of boys between the ages of 0 and 14 underwent orchidopexy. ${ }^{23}$ Because this percentage is higher than the generally accepted estimate of the prevalence of truly non-descended testes (about $1 \%{ }^{4}$ ) we are making efforts to reduce unnecessary orchidopexy.

As we have reason to believe that in the pas the high orchidopexy frequency was related to inaccurate registration of the testes localisation, in some parts of The Netherlands a card for the testes registration was introduced. On this card the localisation of the testes is reg istered by the person who assists the delivery. If the testes are not scrotal at birth the boys are followed up. After introduction of this card in one region the number of operations for orchidopexy was reduced to an acceptable level. ${ }^{3}$

We chose registration soon after birth because the cremaster reflex is still absent at 